\title{
Impacts of varying strengths of intervention measures on secondary outbreaks of COVID-19 in two different regions
}

\author{
Jie Yang · Sanyi Tang $\mathbb{B} \cdot$ Robert A. Cheke
}

Received: 4 September 2020 / Accepted: 5 February 2021 / Published online: 22 February 2021

(C) The Author(s), under exclusive licence to Springer Nature B.V. part of Springer Nature 2021

\begin{abstract}
By March 2020, China and Singapore had achieved remarkable results in the prevention and control of COVID-19, but in April Singapore's outbreak began to deteriorate, while China's remained controlled. Using detailed data from Tianjin, China, and Singapore, a stochastic discrete COVID-19 epidemic model was constructed to depict the impact of the epidemic. Parameter estimation and sensitivity analysis were developed to study the probability of imported cases inducing an outbreak in relation to different prevention and control efforts. Results show that the resumption of work and the re-opening of schools will not lead to an outbreak if the effective reproduction number is lower than 1 and approaches 0 and tracking quarantine measures are strengthened. Once an outbreak occurs, if close contacts can be tracked and quarantined in time, the outbreak will be contained. If work is resumed and schools are re-opened with the effective reproduction number greater than 1 , then it is more likely that a secondary outbreak will be generated. Also, the greater the number of undetected foreign imported cases and the weaker the prevention and con-
\end{abstract}

J. Yang $\cdot$ S. Tang $(\varangle)$

School of Mathematics and Information Science, Shaanxi

Normal University, Xi' an 710062, People's Republic of

China

e-mail: sytang@snnu.edu.cn; sanyitang219@hotmail.com

\section{R. A. Cheke}

Natural Resources Institute, University of Greenwich at Medway, Central Avenue, Chatham Maritime, Chatham, Kent ME4 4TB, UK trol measures, the more serious the epidemic. Therefore, the key to prevention of a second outbreak is to return to work and to re-open schools only after the effective reproduction number is less than 1 for a period, and when tracking quarantine measures have been strengthened. Our model provides a qualitative and quantitative basis for decision-making for the prevention and control of COVID-19 epidemics and the prediction, early warning and risk assessment of secondary outbreaks.

Keywords COVID-19 - Stochastic discrete model · Secondary outbreak - Effective reproduction number . Tracking quarantine measures

\section{Introduction}

After the outbreak of COVID-19 in Wuhan, the infection spread rapidly to all provinces and cities in China. During the early stages, this spread was due to insufficient understanding of the transmission mechanism of the virus, as well as increases in population mobility during the Spring Festival. However, after the lockdown of Wuhan on 23 January 2020 and the strengthening of prevention and control measures in various regions, the epidemic situation in all provinces and autonomous regions was effectively contained and the epidemic situation improved within a short period. Since March 2020, while the epidemic outside China continues to worsen, the epidemic in China has grad- 
ually changed from being dominated by local cases to being dominated by imported cases. At the time of writing (August 2020), the prevention and control and decision-making departments have also adjusted their strategies in a timely manner, from the previous "preventing internal spread and external import" to "preventing external import and internal rebound". Effects of these measures are well reflected by the situation in Tianjin, China. Tianjin had a short outbreak in its early stage and has been well controlled subsequently. Singapore, on the other hand, kept the epidemic under control in the early days, but the situation became serious in April. Tianjin had a permanent population of 15.6 million (2019), and its total area is 1,1966.5 square kilometres [1]. Singapore had a population of 5.7 million (June 2019) and a total area of 724.4 square kilometres (2019) [2]. Both areas are small places, and both did well in the early stages, but later they have become completely opposite to each other, as one has a secondary outbreak, and the other does not. So, a question naturally arises: under what circumstances is there a risk of a secondary outbreak?

In order to answer the above question, the risk of a secondary outbreak induced by imported cases [3], as well as the risk associated with weakening prevention and control measures [3-10], is assessed in this paper to provide early warning for the later development of the epidemic based on model predictions. By integrating multi-source epidemic data from Tianjin and Singapore, the risks of secondary outbreaks in the two regions were systematically studied, and the probability of secondary outbreaks under different management, quarantine and screening measures and different intensity of prevention and control measures analysed. It is concluded that strengthening the management of, and quarantine measures for, inbound travellers are key factors for preventing secondary outbreaks, as is the timely and comprehensive quarantine of close contacts.

\section{Methods}

\subsection{Data collection}

We obtained data on laboratory-confirmed COVID-19 cases in Tianjin and Singapore from the "Health Commission" of Tianjin [11] and the "Ministry of Health" of Singapore [12]. In both areas, very detailed information on each confirmed case has been released. This rigorous contact tracing strategy provided detailed case information on the onset times, first visits to medical facilities, durations of quarantining and confirmations of infection. These data include not only the number of newly reported cases, the cumulative number of confirmed cases reported, the number of recovered cases, the number of deaths, the number of close contacts quarantined and the number of cases medically quarantined and eliminated, but also the date of onset, the date of first medical treatment, the date of laboratory confirmation and date of entry into Tianjin or Singapore (for imported cases). The data were published and analysed anonymously.

Based on the recorded information, we can obtain the daily number of infectious cases with symptoms, quarantined exposed and hospitalized cases, and the daily number of imported cases including exposed, infectious with symptoms, quarantined exposed and hospitalized cases, as shown in Fig. 1. It is worth noting that the data from Tianjin and Singapore are the traced data obtained through detailed data description, so that the likelihood of errors due to random variation is low. Thus, the proposed model could be accurately formulated, and unknown parameters easily estimated by using these source data sets.

\subsection{The model}

Since data on the numbers of COVID-19 infectious cases are now announced daily and considering the randomness of population data, it is appropriate to use a stochastic discrete epidemic model to describe the dynamics of the spread of COVID-19. On the basis of the clinical progression of the disease, epidemiological status of the individuals and intervention measures, we divide the populations into eight classes [13]: susceptible $S(t)$ (individuals who may become infected), exposed $E(t)$ (individuals infected by the virus, in the incubation period), infectious with symptoms $I(t)$ (individuals who have been infected, have illness symptoms and have not yet been quarantined), quarantined susceptible $S_{q}(t)$ (individuals who have close contact with the exposed or infectious, and have been quarantined, but have not been infected), quarantined exposed $E_{q}(t)$ (individuals who have close contact with the exposed or infectious, and have been quarantined, and have been infected), hospitalized $H(t)\left(E_{q}(t)\right.$ or $I(t)$ who have a first medical visit and have been quaran- 
Fig. 1 Data sets for Tianjin from 11 January to 17

March 2020 and for

Singapore from 18 January to 17 March 2020. In A4 and B4, $E_{M}(t)=$ daily numbers of imported exposed cases; $I_{M}(t)=$ daily numbers of imported infectious cases with symptoms; $E_{q M}(t)=$ daily numbers of imported quarantined exposed cases; $H_{M}(t)=$ daily numbers of imported hospitalized cases
(A1)

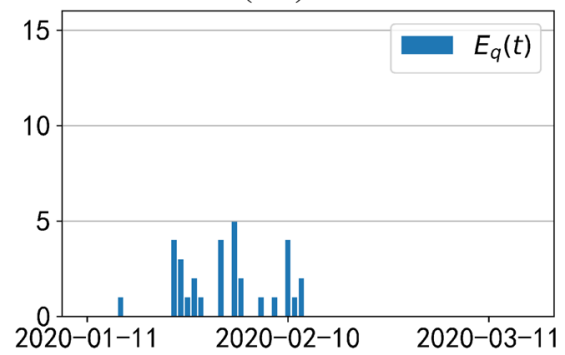

(A2)

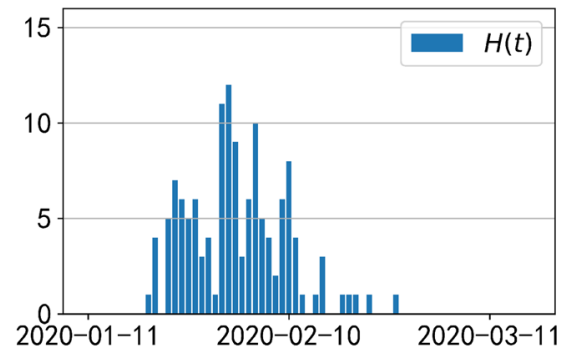

(A3)

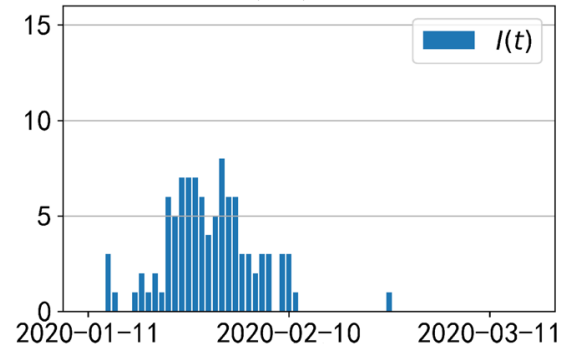

(A4)

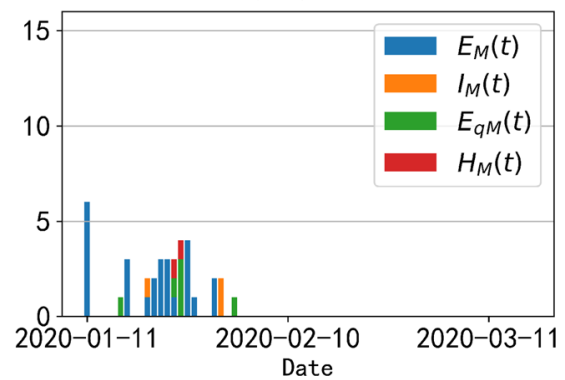

(B1)

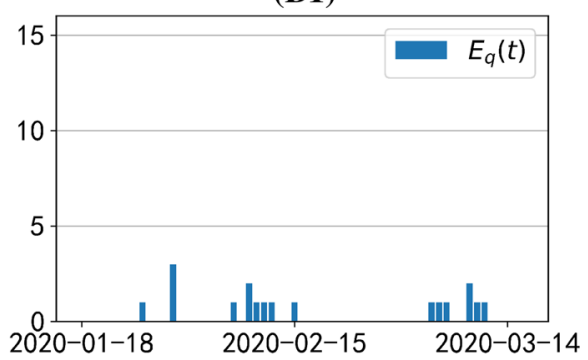

(B2)

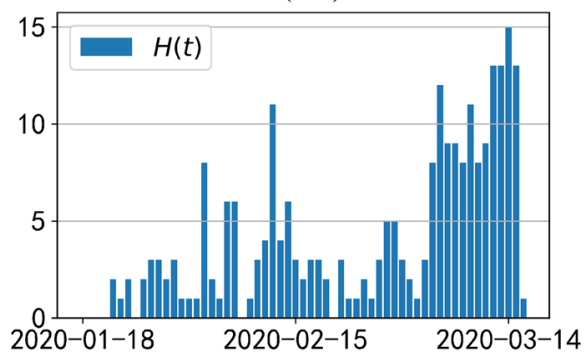

(B3)

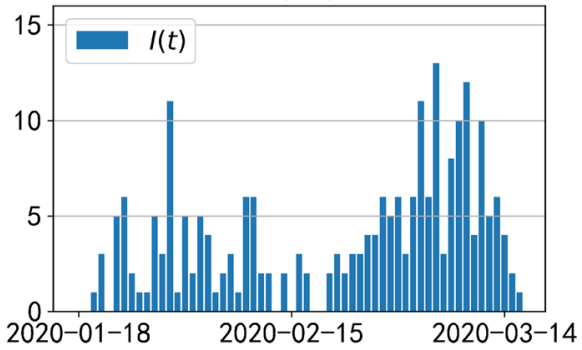

(B4)

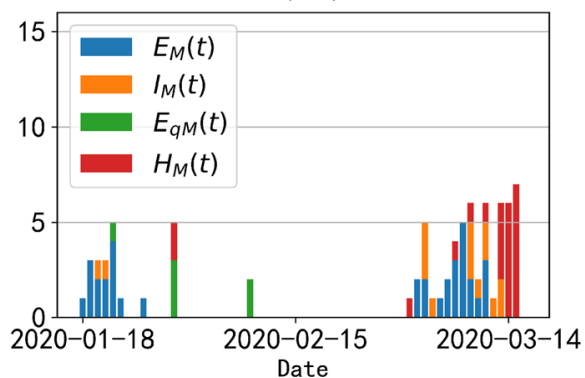

tined), infectious but asymptomatic $A(t)$ (individuals who have been infected, have no illness symptoms and have not yet been quarantined), recovered $R(t)$ (individuals who have recovered from the disease).

The variables $S(t), E(t), I(t), A(t), S_{q}(t), E_{q}(t)$, $H(t)$ and $R(t)$ are the numbers of the individuals in the eight classes at time $t$, respectively. The transfer variables between compartments are denoted by $D_{i j}(t)$. At the same time, the corresponding ran- dom inputs $P_{E}\left(\lambda_{E}\right), P_{I}\left(\lambda_{I}\right), P_{E_{q}}\left(\lambda_{E_{q}}\right), P_{H}\left(\lambda_{H}\right)$ and $P_{A}\left(\lambda_{A}\right)$ with parameters $\lambda_{E}, \lambda_{I}, \lambda_{E_{q}}, \lambda_{H}$ and $\lambda_{A}$ were used to describe the presence of exposed, symptomatic infected persons, quarantined exposed, hospitalized persons and asymptomatic infected persons. The flow diagram is shown in Fig. 2. 


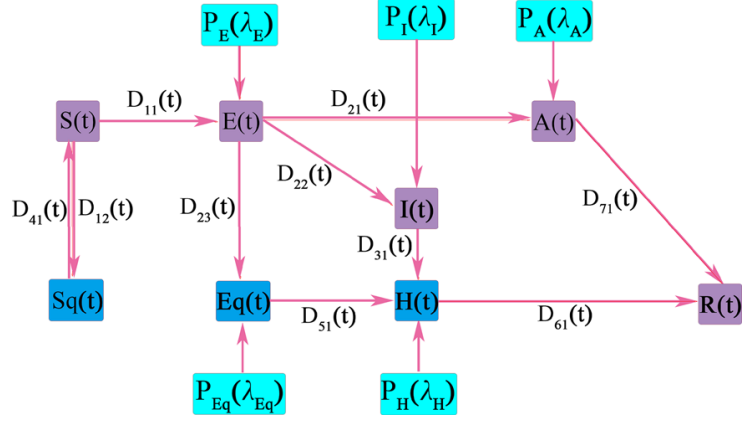

Fig. 2 Diagram of the model adopted in the study for simulating the COVID-19 infection

It follows from [14] that the model equations can be formulated as follows:

$$
\left\{\begin{array}{l}
S_{t+1}=S_{t}-D_{11}(t)-D_{12}(t)+D_{41}(t) \\
E_{t+1}=E_{t}+D_{11}(t)-D_{21}(t)-D_{22}(t)-D_{23}(t)+P_{E}\left(\lambda_{E}\right) \\
I_{\mathrm{t}+1}=I_{t}+D_{22}(t)-D_{31}(t)+P_{I}\left(\lambda_{I}\right) \\
S_{q_{\mathrm{t}+1}}=S_{q_{\mathrm{t}}}+D_{21}(t)-D_{41}(t) \\
E_{q_{\mathrm{t}+1}}=E_{q_{\mathrm{t}}}+D_{23}(t)-D_{51}(t)+P_{E_{q}}\left(\lambda_{E_{q}}\right) \\
H_{t+1}=H_{t}+D_{31}(t)+D_{51}(t)-D_{61}(t)+P_{H}\left(\lambda_{H}\right) \\
A_{\mathrm{t}+1}=A_{t}+D_{21}(t)-D_{71}(t)+P_{A}\left(\lambda_{A}\right) \\
R_{t+1}=R_{t}+D_{61}(t)+D_{71}(t)
\end{array}\right.
$$

where the random variables in (1) can be defined by binomial $\operatorname{Bin}(n, p)$ distributions as follows $[15,16]$ :

$$
\begin{aligned}
& D_{11}(t) \sim \operatorname{Bin}\left(S_{t}, P_{11}(t)\right) \\
& D_{12}(t) \sim \operatorname{Bin}\left(S_{t}, P_{12}(t)\right) \\
& D_{21}(t) \sim \operatorname{Bin}\left(E_{t}, P_{21}(t)\right) \\
& D_{22}(t) \sim \operatorname{Bin}\left(E_{t}, P_{22}(t)\right) \\
& D_{23}(t) \sim \operatorname{Bin}\left(E_{t}, P_{23}(t)\right) \\
& D_{31}(t) \sim \operatorname{Bin}\left(I_{t}, P_{31}(t)\right) \\
& D_{41}(t) \sim \operatorname{Bin}\left(S_{q_{t}}, P_{41}(t)\right. \\
& D_{51}(t) \sim \operatorname{Bin}\left(E_{q_{t}}, P_{51}(t)\right) \\
& D_{61}(t) \sim \operatorname{Bin}\left(H_{t}, P_{61}(t)\right) \\
& D_{71}(t) \sim \operatorname{Bin}\left(A_{t}, P_{71}(t)\right)
\end{aligned}
$$

with probabilities
Note that until 17 March (for Tianjin) and 16 March (for Singapore), there were few deaths, so these had little influence on the total population size. Therefore, we assume that the total number of population $N$ is a constant and let $N=S(t)+E(t)+I(t)+$ $A(t)+S_{q}(t)+E_{q}(t)+H(t)+R(t)$. Considering the asymptomatic cases, let the proportion of asymptomatic infected amongst all infected individuals be $\rho$, and the rate of exposed individuals becoming infectious but asymptomatic be $\sigma_{A}$. Let the rate of exposed individuals becoming infectious with symptoms be $\sigma_{I}$. Also, we assume that the contact number is $c$ and the transmission probability is $\beta$. By contact tracing, we introduced the proportion $q$ of quarantined individuals so that if not infected, the susceptible individuals will move to $S_{q}$ at the rate of $c q(1-\beta)$, but, if infected, the exposed individuals will move to $E_{q}$ at the rate of $q$. The other proportion, $1-q$, including contacts undetected by contact tracing, will become asymptomatic individuals at the rate of $(1-q) \rho \sigma_{A}$, or infectious with symptoms at the rate of $(1-q)(1-\rho) \sigma_{I}$. It is well known that susceptible individuals can be infected by the infectious cases with symptoms as well as asymptomatic persons. Since asymptomatic infected persons have no symptoms of the disease, $\theta$ is set as the correction factor for the transmission probability of asymptomatic infected persons. Table 1 lists all definitions of variables and parameters.

The imported $E(t), I(t), E_{q}(t), H(t)$ and $A(t)$ for each day can be described by Poisson distributions $P_{E}\left(\lambda_{E}\right), P_{I}\left(\lambda_{I}\right), P_{E_{q}}\left(\lambda_{E_{q}}\right), P_{H}\left(\lambda_{H}\right)$ and $P_{A}\left(\lambda_{A}\right)$ with parameters $\lambda_{E}, \lambda_{I}, \lambda_{E_{q}}, \lambda_{H}$ and $\lambda_{A}$, respectively. Therefore, we have [14]

$$
\begin{aligned}
& P_{E}\left(E_{t}=m\right)=\frac{\lambda_{E}{ }^{m} e^{-\lambda_{E}}}{m !}, P_{I}\left(I_{t}=m\right)=\frac{\lambda_{I} e^{-\lambda_{I}}}{m !} \\
& P_{E_{q}}\left(E_{q}=m\right)=\frac{\lambda_{E_{q}} e^{-\lambda_{E_{q}}}}{m !}, P_{H}\left(H_{t}=m\right)=\frac{\lambda_{H}{ }^{m} e^{-\lambda_{H}}}{m !} \\
& P_{A}\left(A_{t}=m\right)=\frac{\lambda_{A} e^{-\lambda_{A}}}{m !}
\end{aligned}
$$

For Tianjin, the contact rate, transmission probability, quarantined rate and diagnosis rate of the model would not be constants after the implementation of the lockdown strategy in Wuhan on 23 January 2020.

$$
\begin{aligned}
& P_{11}(t)=1-\exp \left[-c(t) \beta(t) \frac{\theta A+I}{N}\right], P_{12}(t)=1-\exp \left[-c(t) q(t)(1-\beta(t)) \frac{\theta A+I}{N}\right] \\
& P_{21}(t)=1-\exp \left(-(1-q(t)) \rho \sigma_{A}\right), P_{22}(t)=1-\exp \left(-(1-q(t))(1-\rho) \sigma_{I}\right) \\
& P_{23}(t)=1-\exp (-q(t)), P_{31}(t)=1-\exp \left(-\delta_{I}(t)\right), P_{41}(t)=1-\exp (-\lambda) \\
& P_{51}(t)=1-\exp \left(-\delta_{E_{q}}\right), P_{61}(t)=1-\exp \left(-\gamma_{H}\right), P_{71}(t)=1-\exp \left(-\gamma_{A}\right)
\end{aligned}
$$


We set $T_{0}$ to be 22 January 2020 . The contact number $c(t)$ and transmission probability $\beta(t)$ are modelled as decreasing functions over time $t$, given by [14] (district)-level health authorities should organize and coordinate the tracing and management of close contacts. Such close contacts of confirmed cases were

$c(t)=\left\{\begin{array}{l}c_{0}, \text { before } 23 \text { January } 2020 \\ \left(c_{0}-c_{b}\right) \mathrm{e}^{-r_{1}\left(t-T_{0}\right)}+c_{b}, \text { from } 23 \text { January to } 17 \text { March } 2020\end{array}\right.$

$\beta(t)=\left\{\begin{array}{l}\beta_{0}, \text { before } 23 \text { January } 2020 \\ \left(\beta_{0}-\beta_{a}\right) \mathrm{e}^{-r_{4}\left(t-T_{0}\right)}+\beta_{a}, \text { from } 23 \text { January to } 17 \text { March } 2020\end{array}\right.$

Similarly, to describe enhanced contact tracing, we define $q(t)$ as an increasing function of time $t$, as [14] placed under home or centralized quarantine for medical observation for 14 days after the last contact with

$q(t)=\left\{\begin{array}{l}q_{0}, \text { before } 23 \text { January } 2020 \\ \left(q_{0}-q_{m}\right) \mathrm{e}^{-r_{2}\left(t-T_{0}\right)}+q_{m}, \text { from } 23 \text { January to } 17 \text { March } 2020\end{array}\right.$

We also set the transition rate $\delta_{I(t)}$ as an increasing function with respect to time $t$; thus, the detection period $\frac{1}{\delta_{I(t)}}$ is a decreasing function of $t$ with the following form [14]

$\frac{1}{\delta_{I}(t)}=\left\{\begin{array}{l}\frac{1}{\delta_{I_{0}}}, \text { before } 23 \text { January } 2020 \\ \left(\frac{1}{\delta_{I_{0}}}-\frac{1}{\delta_{I_{f}}}\right) \mathrm{e}^{-r_{3}\left(t-T_{0}\right)}+\frac{1}{\delta_{I_{f}}}, \text { from } 23 \text { January to } 17 \text { March } 2020\end{array}\right.$ a case on 28 January. In Singapore, the "Ministry of Health" [12] issued a press release announcing that the health status of all close contacts would be closely monitored, and as a precautionary measure, they would be quarantined for 14 days from their last exposure to the patient when the first COVID-19 patient was con-
For Singapore, according to the Ministry of Health [12], it was aware of the cluster of severe pneumonia cases in Wuhan city, Hubei Province, China, and was monitoring the situation closely on 2 January 2020. Also, the Ministry reported that from the evening of 3 January 2020 temperature screening would be implemented at Changi Airport for inbound travellers arriving on flights from Wuhan, and suspected cases would be referred to hospitals for further assessment. Health advisory posters for all travellers would be put up at Changi Airport, and health advisory information would be provided to all inbound travellers on flights from Wuhan. Clearly, Singapore had been aware of the seriousness of COVID-19 since 2 January 2020 and has always attached great importance to the development of the epidemic, so we can treat the above four parameters as constants.

\subsection{Simulation}

The "National Health Commission" of the People's Republic of China [17] had issued a notice that county firmed on 23 January. The information above means that the quarantined susceptible will be removed after 14 days, obviously, so we set $\lambda=1 / 14$. Estimation of the asymptomatic ratio, the percentage of carriers with no symptoms, will improve understanding of COVID-19 transmission and the spectrum of disease it causes, providing insight into epidemic spread. For this, we used information on Japanese nationals who were evacuated from Wuhan, China, on charter flights and employing a Bayes theorem, we estimated the asymptomatic ratio amongst evacuees to be $30.8 \%$ [18], thus setting $\rho=$ 0.308 . For Tianjin, using data extracted from the Health Commission of Tianjin from 11 January to 17 March 2020 the values of some parameters can be obtained by a simple analysis of the recorded data. The mean duration from illness onset to first medical visit before 23 January was 5 days; therefore, we set $\delta_{I_{0}}=1 / 5$. The mean duration from quarantined exposed to first medical visit was 4.75 days; therefore, we set $\delta_{E_{q}}=$ 1/4.75. Obviously, $q_{0}=0$. Similarly, for Singapore, we used data extracted from the Ministry of Health from 
Fig. 3 Model fitting for the daily number of quarantined exposed, infectious with symptoms and hospitalized cases in Tianjin (A1-A3) and Singapore (B1-B3)
(A1)

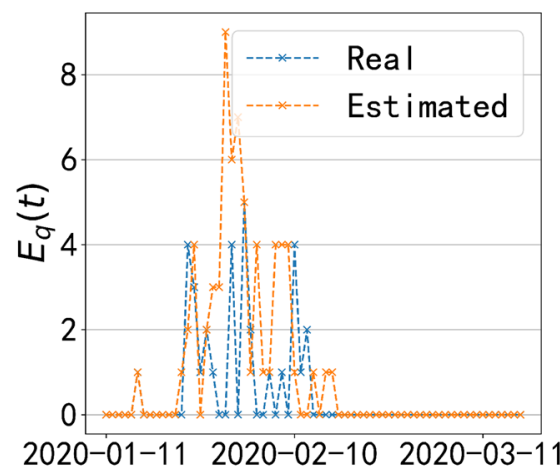

(A2)

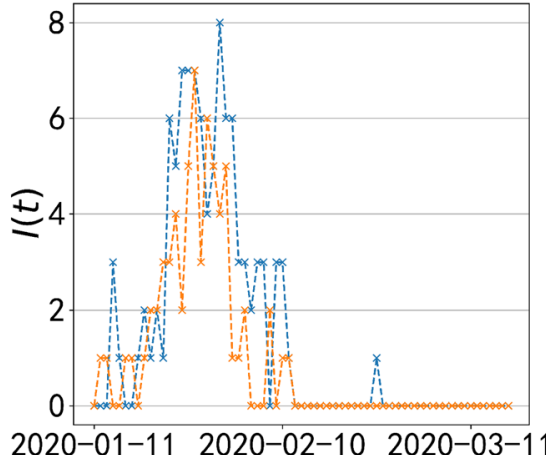

(A3)

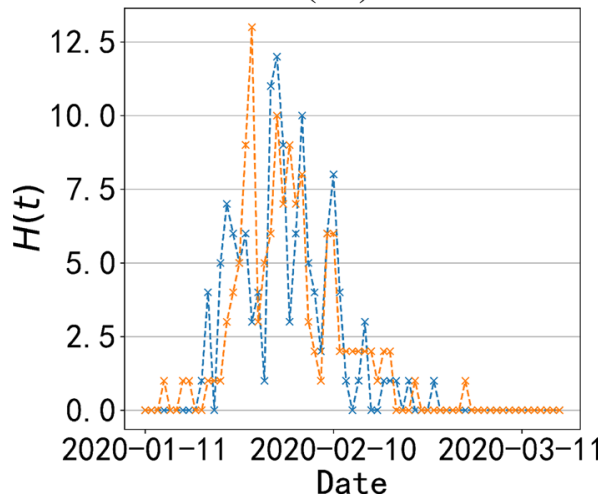

(B1)

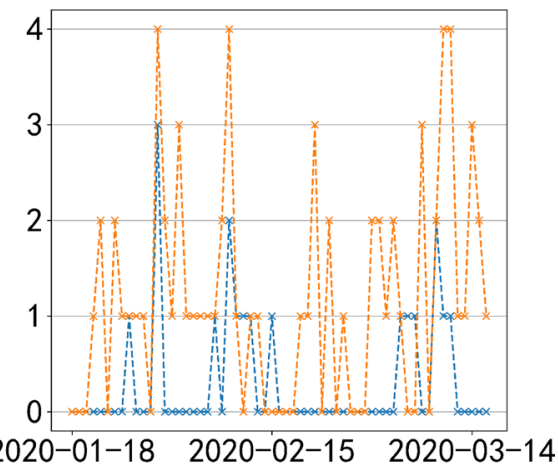

(B2)

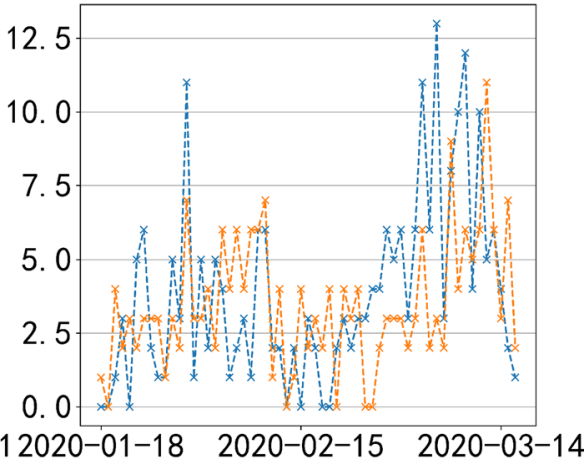

(B3)

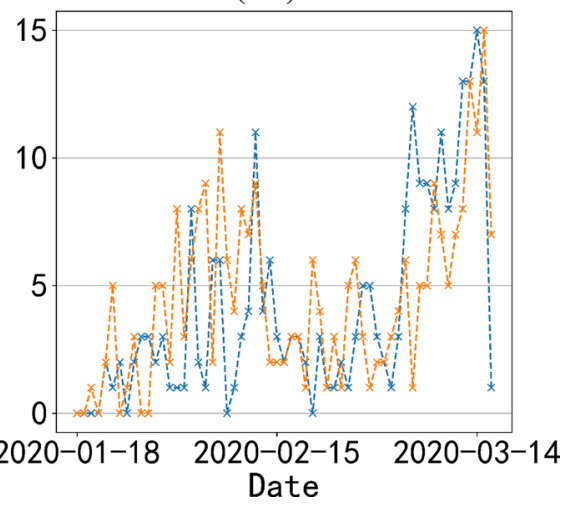

18 January to 16 March 2020 getting $\delta_{I_{0}}=1 / 4.75$ and $\delta_{E_{q}}=1 / 5.67$.

\section{Main results}

\subsection{Parameter estimation and model fitting}

By fitting data on the daily number of quarantined exposed, infectious with symptoms and hospitalized cases, we obtained the estimated values of other parameters. To do so, we utilized the nonlinear least squares (NLES) method in MATLAB to fit the aforementioned real data sets which correspond to the model solution time series, i.e. $D_{23}(t)+P_{E_{q}}\left(\lambda_{E_{q}}\right), D_{22}(t)+P_{I}\left(\lambda_{I}\right)$, and $D_{31}(t)+D_{51(t)}+P_{H}\left(\lambda_{H}\right)$, as shown in Fig. 3. The estimated parameter values are listed in Table 1. 
Table 1 Initial conditions and parameter values for model (1)

\begin{tabular}{|c|c|c|c|c|c|}
\hline \multicolumn{2}{|c|}{ Initial conditions } & \multirow{2}{*}{$\begin{array}{l}\text { Description } \\
\text { Initial susceptible population }\end{array}$} & \multirow{2}{*}{$\begin{array}{l}\text { Value } \\
\text { Tianjin } \\
1.56 \times 10^{7}\end{array}$} & \multirow{2}{*}{$\frac{\text { Singapore }}{5.7 \times 10^{6}}$} & \multirow{2}{*}{$\begin{array}{l}\text { Reference } \\
{[1,2]}\end{array}$} \\
\hline$S(0)$ & & & & & \\
\hline$E(0)$ & & Initial exposed population & 5 & 6 & Fitting \\
\hline$I(0)$ & & Initial infected population with symptoms & 0 & 0 & Data \\
\hline$A(0)$ & & Initial infected population but asymptomatic & 0 & 0 & Data \\
\hline$S_{q}(0)$ & & Initial quarantined susceptible population & 0 & 0 & Data \\
\hline$E_{q}(0)$ & & Initial quarantined exposed population & 0 & 0 & Data \\
\hline$H(0)$ & & Initial hospitalized population & 0 & 0 & Data \\
\hline$R(0)$ & & Initial recovered population & 0 & 0 & Data \\
\hline \multicolumn{2}{|c|}{ Parameters } & Description & Value & & Reference \\
\hline & & & Tianjin & Singapore & \\
\hline \multicolumn{2}{|l|}{$\rho$} & Proportion of asymptomatic infected individuals & 0.308 & & {$[18]$} \\
\hline \multicolumn{2}{|l|}{$\theta$} & $\begin{array}{l}\text { Correction factor for transmission probability of } \\
\text { asymptomatic infected individuals }\end{array}$ & 0.1 & 0.1 & Fitting \\
\hline \multicolumn{2}{|l|}{$\sigma_{A}$} & $\begin{array}{l}\text { Transition rate of exposed individuals to the } \\
\text { symptomatic infected individuals }\end{array}$ & 0.1 & 0.1 & Fitting \\
\hline \multicolumn{2}{|l|}{$\sigma_{I}$} & $\begin{array}{l}\text { Transition rate of exposed individuals to the } \\
\text { asymptomatic infected individuals }\end{array}$ & 0.1 & 0.5 & Fitting \\
\hline \multicolumn{2}{|l|}{$\lambda$} & $\begin{array}{l}\text { Rate at which the quarantined uninfected contacts were } \\
\text { released into the wider community }\end{array}$ & $1 / 14$ & & {$[12,17]$} \\
\hline \multirow[t]{3}{*}{$c(t)$} & $c_{0}$ & Contact number at the initial time & 20 & 6 & Fitting \\
\hline & $c_{b}$ & Minimum contact number under the current control strategies & 8 & - & Fitting \\
\hline & $r_{1}$ & Exponential decreasing rate of contact number & 0.3 & - & Fitting \\
\hline \multirow[t]{3}{*}{$q(t)$} & $q_{0}$ & Quarantined rate of close contacts at the initial time & 0 & 0.08 (Fit) & Data \\
\hline & $q_{m}$ & Maximum quarantined rate & 0.6 & - & Fitting \\
\hline & $r_{2}$ & Exponential increasing rate of quarantined rate & 0.02 & - & Fitting \\
\hline \multirow[t]{3}{*}{$\delta_{I}(t)$} & $\delta_{I_{0}}$ & $\begin{array}{l}\text { Initial diagnosis rate of symptomatic infected } \\
\text { individuals to the hospitalized class }\end{array}$ & $1 / 5$ & $1 / 4.75$ & Data \\
\hline & $\delta_{I_{f}}$ & Fastest diagnosis rate & 1 & - & Fitting \\
\hline & $r_{3}$ & Exponential increasing rate of diagnosis rate & 0.5 & - & Fitting \\
\hline \multirow[t]{3}{*}{$\beta(t)$} & $\beta_{0}$ & Initial transmission probability & 0.1 & 0.05 & Fitting \\
\hline & $\beta_{a}$ & Minimum transmission probability & 0.002 & - & Fitting \\
\hline & $r_{4}$ & Exponential decreasing rate of transmission probability & 0.2 & - & Fitting \\
\hline \multicolumn{2}{|l|}{$\delta_{E_{q}}$} & $\begin{array}{l}\text { Diagnosis rate of quarantined exposed individuals to the } \\
\text { hospitalized class }\end{array}$ & $1 / 4.75$ & $1 / 5.67$ & Data \\
\hline \multicolumn{2}{|l|}{$\gamma_{H}$} & Recovery rate of hospitalized individuals to the recovered class & 0.05 & 0.05 & Fitting \\
\hline \multicolumn{2}{|l|}{$\gamma_{A}$} & $\begin{array}{l}\text { Recovery rate of symptomatic infected individuals to the } \\
\text { recovered class }\end{array}$ & 0.08 & 0.1 & Fitting \\
\hline
\end{tabular}


(A)

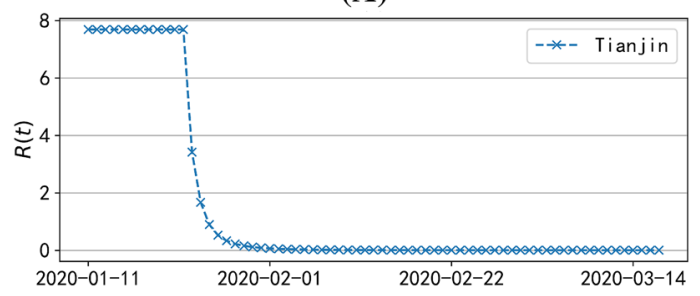

(B)

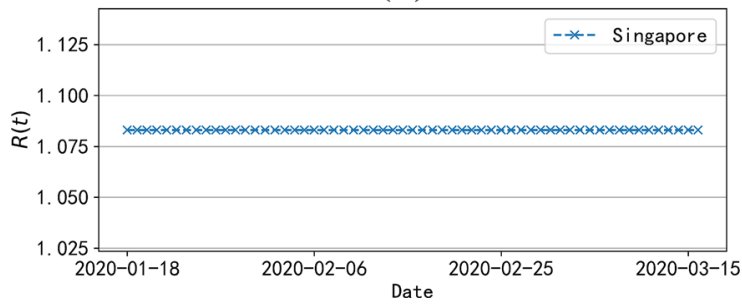

Fig. 4 The effective reproduction number $R(t)$ in Tianjin during the period 11 January to 17 March 2020 (a) and for Singapore from 18 January to 16 March 2020 (b)

\subsection{Effective reproduction number}

For an epidemic model, it is important to estimate the effective reproduction number. The effective reproduction number, $R$ (defined as the average number of secondary cases that one index case generates over the course of its infectious period), is a useful measure of transmissibility and can be estimated over time $(R(t))$ through the course of an epidemic [19]. $R(t)$ shows time-dependent variation due to the decline in susceptible individuals (intrinsic factors) and the implementation of control measures (extrinsic factors) [20]. By using the next-generation matrix approach for discretetime epidemic models [21,22], we get the effective reproduction number for system (1) as

$R=\frac{c \beta\left(\rho \theta \delta_{I} \sigma_{A} q+\sigma_{I}(1-q) \gamma_{A} \rho-\rho \theta \delta_{I} \sigma_{A}-\sigma_{I}(1-q) \gamma_{A}\right)}{\left(\sigma_{I}(1-q) \rho+\rho \sigma_{A} q-\sigma_{I}(1-q)-q-\rho \sigma_{A}\right) \delta_{I} \gamma_{A}}$

According to the parameter values given in Table 1, the effective reproduction numbers in Tianjin and Singapore are shown in Fig. 4.

As can be seen from Fig. 4, the effective reproduction number for Tianjin was close to 8 from 11 to 22 January, and declined rapidly from 23 January, approached 0 from 31 January, and maintained this state until 17 March. That is, the outbreak in Tianjin was very serious at the beginning, but it was soon effectively controlled and kept under strict control, providing good condi- tions for the resumption of work and the re-opening of schools.

The effective reproduction number for Singapore from 18 January to 16 March was a constant relatively low value (1.083), indicating that the outbreak was well contained from the start. But it is greater than 1, meaning that there was a risk of an outbreak, and if Singapore had chosen to resume work and re-open schools in this state, the risk of a second outbreak will be high.

It can be seen from formula (2) of the effective reproduction number that it can reflect the intensity of control measures in a region. According to Table 1, the quarantined rate of Singapore was only 0.08, while the quarantined rate of Tianjin was 0 at the beginning, but it rose rapidly from 23 January, and the quarantined rate of Tianjin was obviously much higher than that for Singapore in the later period. Therefore, Singapore's high effective reproduction number was associated with its low quarantined rate. In other words, Singapore's screening of close contacts was not as strong as Tianjin's, and its low level of screening could lead to a second outbreak in the future.

\section{Impact of foreign imported cases and prevention and control measures on COVID-19 outbreaks}

\subsection{Tianjin}

For Tianjin, according to the current data, COVID-19 has been well controlled, and there has been a lot of detailed data released, which can clearly tell us whether the cases are asymptomatic infected persons $(A)$ and whether they have symptoms or not when they are imported ( $I$ or $E$ ). Considering the practical situation, it is known that the cases who are in the symptomatic state when they are imported will be easily detected (all cases in compartment $I$ enter directly into compartment $H$, and there are no cases in compartment $I$ ), generally, so the main consideration is that asymptomatic infections $(A)$ and imported cases during the incubation period $(E)$ may not be detected. Here, parameter $k(k=0.1,0.3,0.6)$ is introduced as the proportion of undetected infected people as a measure of the imported cases who were not detected when they arrived. The proportion $k$ of cases in compartments $A$ and $E$ will stay the same, and the rest of the cases which have been detected will enter compartment $E_{q}$. 
(A1)

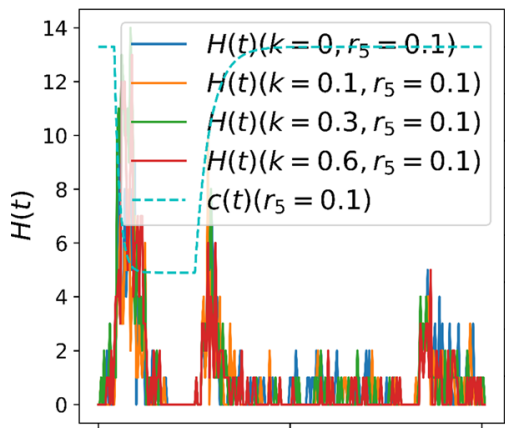

2020-01-11
(B1)

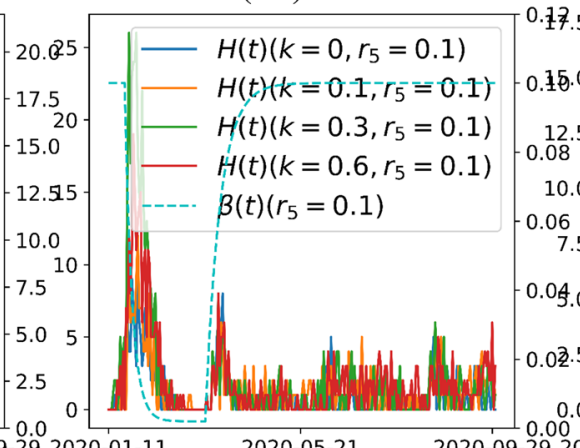

(B2)
(C1)

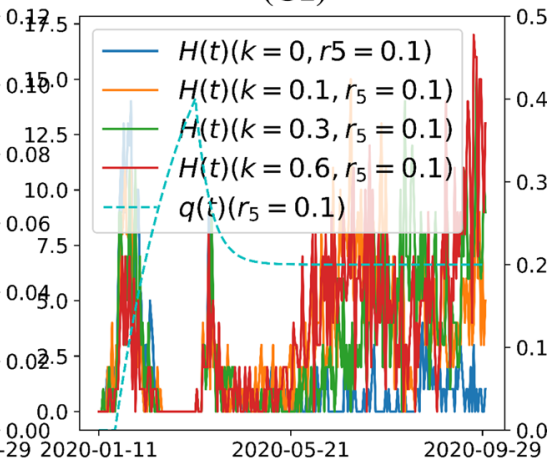

(A2)
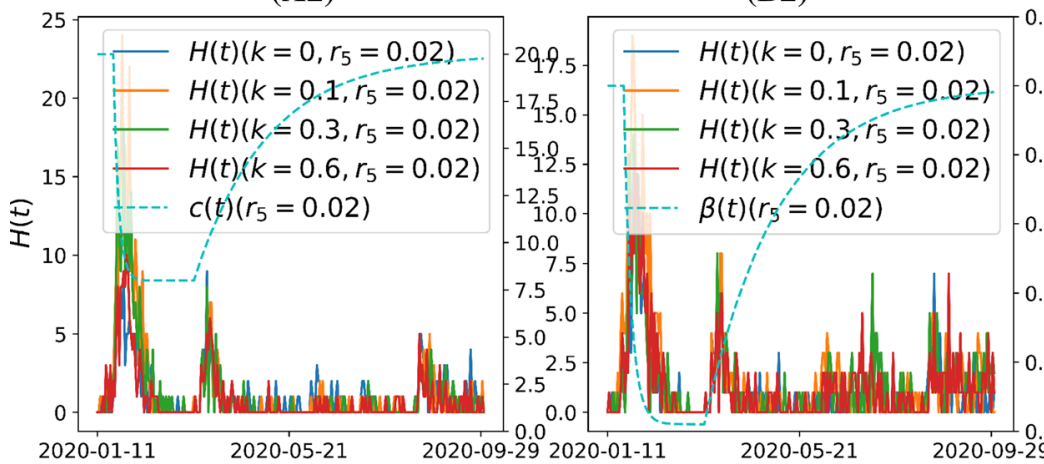

(C2)

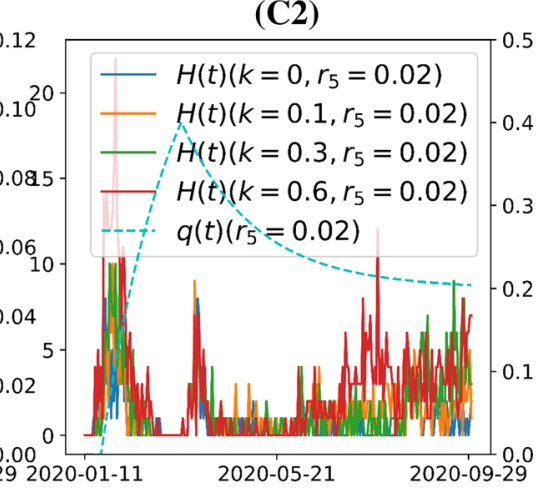

(A3)

(B3)

(C3)
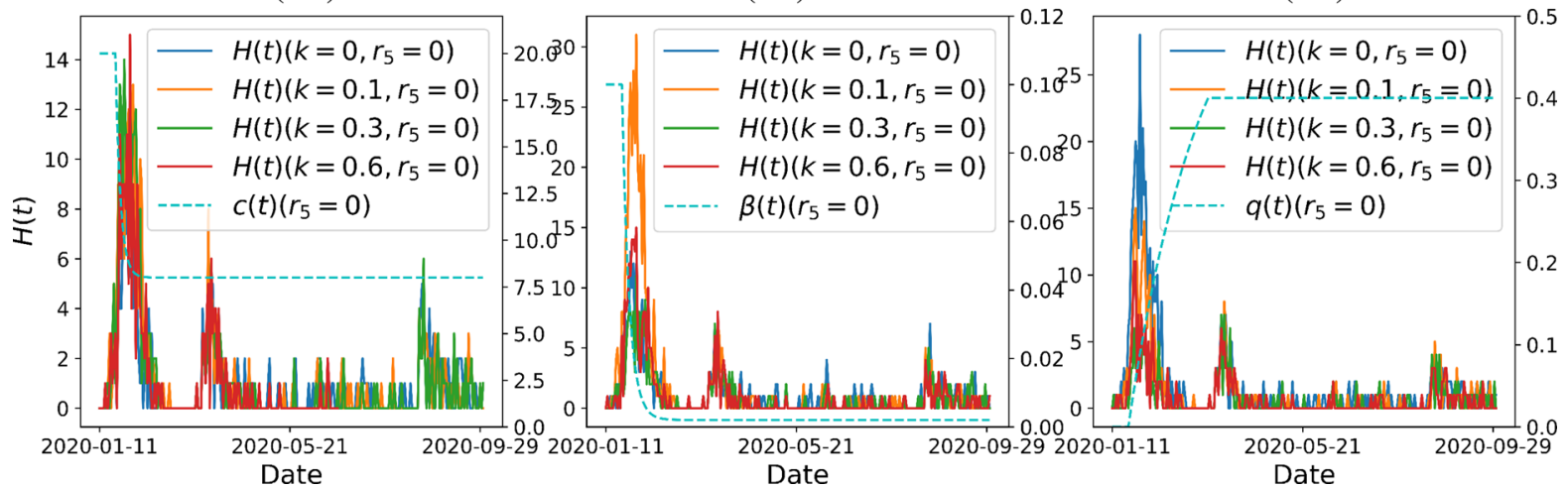

Fig. 5 The effects of foreign imported cases and lifting the prevention and control measures on the possibility of a secondary outbreak of COVID-19 in Tianjin. A1-A3 The hospitalized cases based on fully lifting the prevention and control measures about $c$ with all combinations of $k$ and $r_{5}$ and the corresponding $c(t)$. B1-B3 The hospitalized cases based on both fully lifting the prevention and control measures about $c$ and $\beta$ with all combinations of $k$ and $r_{5}$ and the corresponding $\beta(t)$. C1-C3 The hospitalized cases based on both fully lifting the prevention and control measures about $c$ and $\beta$ and 50\% lifting the prevention and control measures about $q$ with all combinations of $k$ and $r_{5}$ and the corresponding $q(t)$ 
On the other hand, the resumption of work and reopening of schools has made people less concerned about the epidemic. However, the effects of the prevention and control measures reflected by the contact faster the corresponding growth or decrease rate is. In the case of the weakest prevention and control measures described above, the corresponding $c(t), \beta(t)$ and $q(t)$ in model (1) from 11 January to 8 June are as follows:

$c(t)=\left\{\begin{array}{l}c_{0}, \text { before 23 January } 2020 \\ \left(c_{0}-c_{b}\right) \mathrm{e}^{-r_{1}\left(t-T_{0}\right)}+c_{b}, \text { from } 23 \text { January to } 17 \text { March } 2020 \\ \left(c_{01}-c_{b 1}\right) \mathrm{e}^{-r_{5}\left(t-T_{1}\right)}+c_{b 1}, \text { from } 18 \text { March to } 8 \text { June } 2020\end{array}\right.$

$\beta(t)=\left\{\begin{array}{l}\beta_{0}, \text { before } 23 \text { January } 2020 \\ \left(\beta_{0}-\beta_{a}\right) \mathrm{e}^{-r_{4}\left(t-T_{0}\right)}+\beta_{a}, \text { from } 23 \text { January to } 17 \text { March } 2020 \\ \left(\beta_{01}-\beta_{a 1}\right) \mathrm{e}^{-r_{5}\left(t-T_{1}\right)}+\beta_{a 1}, \text { from March } 18 \text { to } 8 \text { June } 2020\end{array}\right.$

$q(t)=\left\{\begin{array}{l}q_{0}, \text { before } 23 \text { January } 2020 \\ \left(q_{0}-q_{m}\right) \mathrm{e}^{-r_{2}\left(t-T_{0}\right)}+q_{m}, \text { from } 23 \text { January to } 17 \text { March } 2020 \\ \left(q_{01}-q_{m 1}\right) \mathrm{e}^{-r_{5}\left(t-T_{1}\right)}+q_{m 1}, \text { from } 18 \text { March to } 8 \text { June } 2020\end{array}\right.$

number $c$, the transmission probability $\beta$ and the quarantined rate $q$ are different [4,23-25], and people attach different importance to them. Amongst the public, once the emphasis on the epidemic situation drops, it will generally be reflected in an increase in $c$. If the emphasis is lower, there may be less emphasis on wearing masks and other precautionary measures, leading to an increase in $\beta$. At the lowest level of emphasis, there is a high level of confidence that there are no local cases, and that the symptoms of a COVID-19 case are not reported in time, leading to reduced tracing and quarantine of close contacts, thus leading to the decrease of $q$.

Interventions of different degrees have different effects [26,27], so three different degrees of the weakening of prevention and control measures are given: (1) only $c$ increases ( $\beta$ and $q$ maintain the original trend); (2) both $c$ and $\beta$ increase ( $q$ maintains the original trend) and (3) both $c$ and $\beta$ increase and $q$ decreases. To better study the impact of changes in $c, \beta$ and $q$ on the epidemic we, firstly, suppose that the slackening of the prevention and control measures began on 18 March. Secondly, suppose that if $c$ and $\beta$ become larger (i.e. fully lifting the prevention and control measures about $c$ and $\beta$ ), their maximum values can reach the value before the Wuhan blockade. Finally, it is assumed that if $q$ decreases, its lowest value can reach half of the highest quarantined rate in Tianjin, that is, $50 \%$ lifting the prevention and control measures about $q$. A new parameter $r_{5}$ is introduced to characterize the exponential increasing rate of $c$ and $\beta$ and the exponential decreasing rate of $q$ uniformly. The larger $r_{5}$ is, the
The risk of a secondary outbreak in Tianjin under all combinations of $k$ and $r_{5}$ was considered based on the above three degrees of neglect (as shown in Fig. 5). In particular, if the value of $H(t)$ is always below 20 after March 18, then there is no secondary outbreak in Tianjin, and if $H(t)$ is greater than 20 at some time, then there is a secondary outbreak in Tianjin.

As can be seen from Fig. 5, there is no risk of a secondary outbreak. In particular, when all the prevention and control measures are relaxed to the weakest point (as shown in Fig. 5C1), that is, both fully lifting the prevention and control measures about $c$ and $\beta$ and $50 \%$ lifting the prevention and control measures about

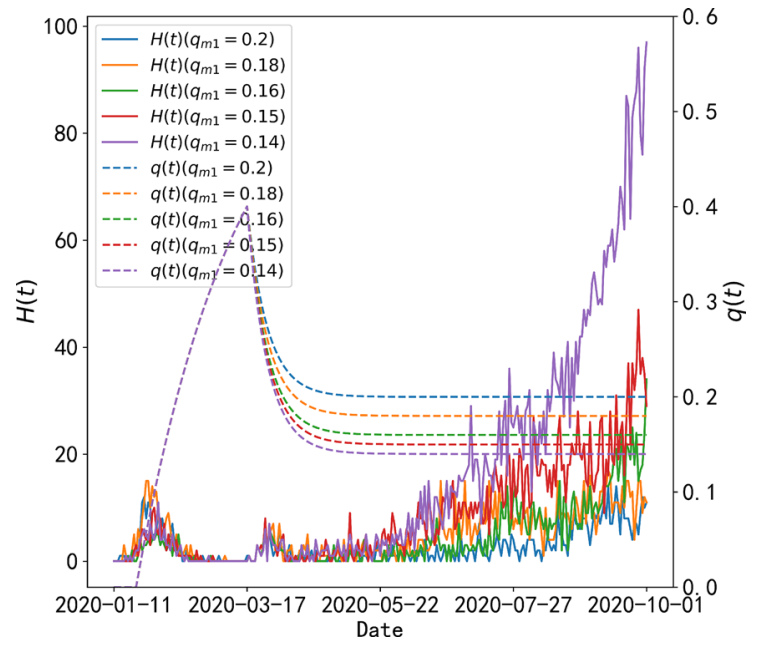

Fig. 6 Impacts of lifting the prevention and control measures about $q$ on a potential secondary outbreak of COVID-19 with $k=0.6$ and $r_{5}=0.1$ in Tianjin 

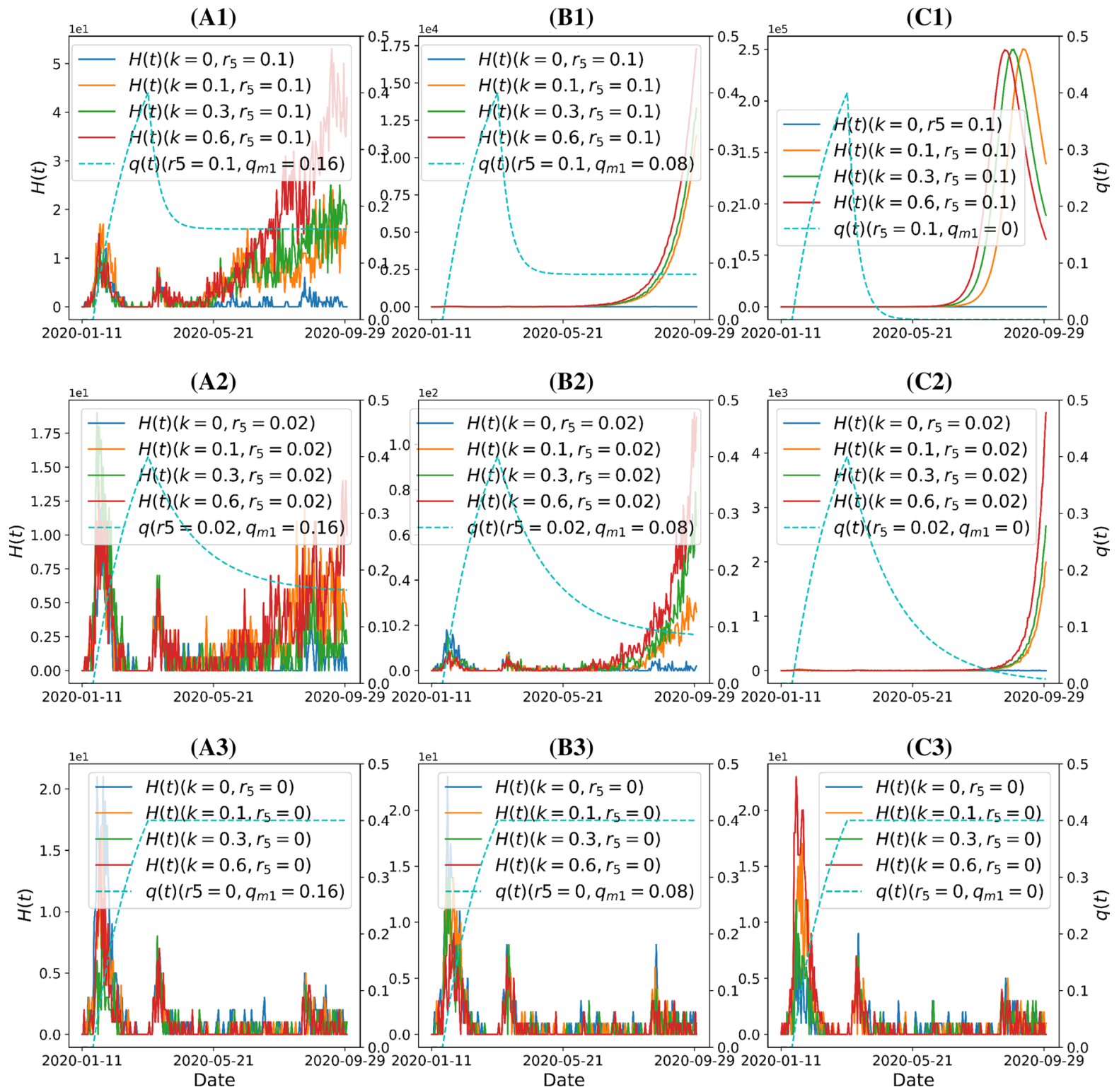

Fig. 7 The effects of foreign imported cases and lifting the prevention and control measures about $q$ on a potential secondary outbreak of COVID-19 in Tianjin. The hospitalized cases based on both fully lifting the prevention and control measures about $c$ and $\beta$, and 60\% (A1-A3), 80\% (B1-B3) and fully (C1-C3) lifting the prevention and control measures about $q$ with all combinations of $k$ and $r_{5}$ and the corresponding $q(t)$ $q$, the possibility of a secondary outbreak is almost 0 . Although the value of $q$ is already very low, it is obvious that $q$ could be lower. Therefore, to find the minimal $q_{m 1}$ resulting in a secondary outbreak of COVID-19 in Tianjin, we continuously reduced $q_{m 1}$ under the circumstance of $k=0.6$ and $r_{5}=0.1$, the results of which are shown in Fig. 6.
It follows from Fig. 6 that with a high quarantined rate involving intensive tracking and quarantine measures, even if both fully lifting the prevention and control measures about $c$ and $\beta$ and some of the imported cases have gone undetected, the secondary outbreak of the epidemic in Tianjin cannot occur. However, when $q_{m 1}$ drops to a threshold $\left(q_{m 1}=0.16\right)$, there will be a 


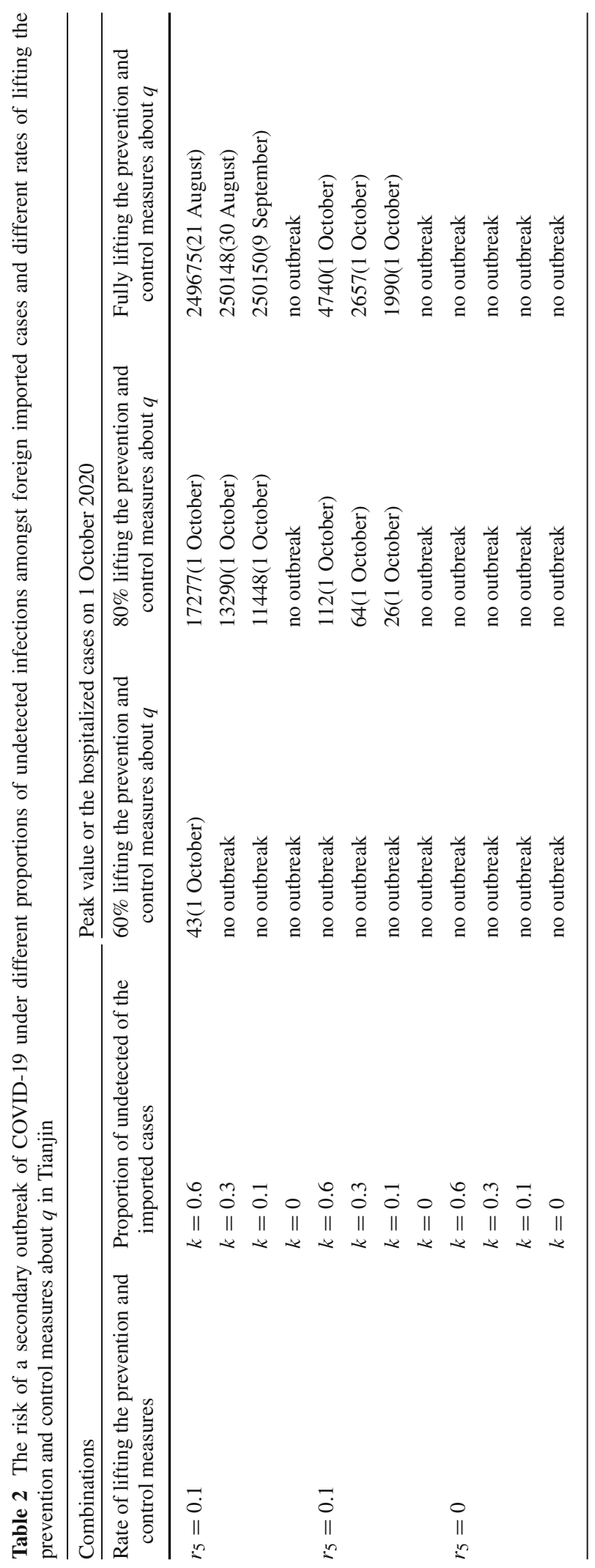


secondary outbreak, and the lower $q_{m 1}$ drops, the more serious the epidemic becomes, which emphasizes the importance of the quarantined rate. Therefore, in order to understand the effect of the quarantined rate on secondary outbreaks more fully, the effects of different rates of lifting the prevention and control measures $\left(r_{5}\right)$ and the proportion $(k)$ of undetected imported cases were investigated for variations of $q_{m 1}$, as shown in Fig. 7 and Table 2. According to model (1) and Table 1 , it is easy to calculate that $q_{3 / 17}=0.400277$, so $q_{m 1}=0.16, q_{m 1}=0.08$ and $q_{m 1}=0$, respectively, which indicate that $60 \%, 80 \%$ and fully lifting the prevention and control measures about $q$ have been implemented.

It is clear according to Fig. 7B1, B2, C1, C2 and Table 2 that even with the same intensity of lifting the prevention and control measures about quarantined rate $q$, different lifting speeds result in huge variation in the final outbreak size (about 100 times). It follows from Fig. 7C1 that under our assumption above, the largest secondary outbreak in Tianjin would have reached about 250,000 people in August and September.

The results shown in Figs. 5 and 7 indicate that fully lifting the prevention and control measures about contact number $c$ and transmission possibility $\beta$ cannot result in a secondary outbreak, while more than $60 \%$ lifting the prevention and control measures about quarantined rate $q$ will have a risk of secondary outbreak. Due to the effect of quarantine-related prevention and control measures on the socioeconomics, it is advisable to keep a relative low quarantined rate, i.e. 0.16 at all times.

According to the Beijing Municipal Health Commission [28], there was a secondary outbreak in June, and the outbreak was quickly brought under control. Rigorous and meticulous screening of close contacts was carried out. So, it is speculated that the rapid control of the epidemic is related to the higher quarantined rate. In order to verify the above speculation, the data for Tianjin were used for simulation. On the basis of Fig. $7 \mathrm{C} 1$, it is assumed that the quarantined rate would increase rapidly starting from 10 May.

According to Fig. 8, when COVID-19 cases occur, the outbreak can be quickly contained as long as the close contacts of the cases can be tracked and quarantined in a timely manner (i.e. the quarantined rate is high).

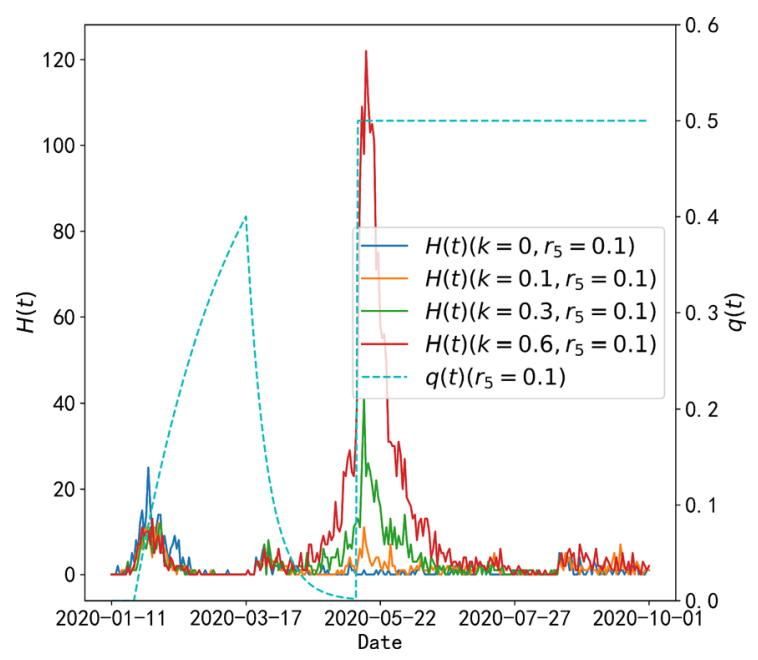

Fig. 8 Impact of the quarantined rate on the secondary outbreak of COVID-19 in Tianjin. The hospitalized cases based on both fully lifting the prevention and control measures about $c$ and $\beta$ with $r_{5}=0.1$ from 18 March to 8 June and on $q$ (decreased from 18 March to May 10 and directly increased to 0.5 after 10 May) and the corresponding $q(t)$

\subsection{Singapore}

For Singapore, it can be seen from the Ministry of Health [12] that since 17 March, only the numbers of confirmed and imported cases have been published, with no detailed information. In order to better simulate the status of imported cases, some assumptions were made. We assumed that some of the confirmed imported cases were in the incubation period when they arrived and that some were symptomatic. It is also known from the data before 17 March that approximately $50 \%$ of the imported cases were in the incubation period at the time of import, so it is assumed that $50 \%$ of the imported cases were in the incubation period and the rest were symptomatic. According to the detailed data before 17 March, almost all of the imported cases with symptoms were quarantined and admitted to hospital on the same day; thus, we guessed that $50 \%$ of the imported cases arrived on the day of confirmation of their infections and so directly entered compartment $H$, while compartment $I$ had no additions. As for confirmed cases imported during the incubation period, it is known that the average time from the importation to entering the compartment $H$ before 17 March was about 5 days, so it was assumed that the time follows a Poisson distribution with an average of 5, thus generating the imported date of each case, which is the date of the importation to 
the compartment $E$. It is known that the data collected only included imported confirmed cases so it is impossible to measure the number of imported asymptomatic infected persons. However, according to the literature [18], asymptomatic infected persons may account for $30.8 \%$ of all infected persons, so the date of importation of asymptomatic infected persons can be summarized as $A=H /(1-0.308) \times 0.308$. Based on the above hypothesis, we obtained the dates of the imported cases into the compartments $A, I, H$ and $E$, respectively. As with the Tianjin data, we measured the undetected ratio with parameter $k$. On the other hand, given the outbreak of COVID-19 in Singapore after April, it may be assumed that from 17 March, the contact number $c$ and transmission probability $\beta$ began to increase (up to twice as high), and the quarantined rate $q$ began to decrease, the lowest of which will be half of the value before. The parameter $r_{5}$ is introduced to characterize the exponentially increasing rate of $c(t)$ and $\beta(t)$, as well as the exponentially decreasing rate of $q(t)$, consistent with Tianjin. The corresponding $c(t), \beta(t)$ and $q(t)$ in model (1) from 18 January to 12 June are as follows: demic also increases. When the previous prevention and control measures are maintained $\left(r_{5}=0\right.$, as shown in Fig. 9A3, B3, C3, which depict the same situation), if no cases are undetected, the epidemic will still have a small secondary outbreak. This means that the secondary outbreak was caused by local cases, indicating that some close contacts or asymptomatic infected persons may have been missed in Singapore in the early stage. In this state, it is not enough to only maintain the prevention and control measures in the early stage, and the prevention and control measures need to be further strengthened to avoid the secondary outbreak.

If the prevention and control measures had weakened $\left(r_{5} \neq 0\right.$, as shown in Fig. 9A1, A2, B1, B2, $\mathrm{C} 1, \mathrm{C} 2$ ), the outbreak would seriously increase to the point of being almost completely out of control, and the higher the rate of lifting the prevention and control measures, the more serious the epidemic. Also, due to lifting the prevention and control measures, the influence of the undetected imported cases would be almost negligible. According to Fig. 9A2, A3, if contact number increased by $100 \%$, even if with the lower rate of lifting the prevention and control measures $\left(r_{5}=0.02\right)$,

$c(t)=\left\{\begin{array}{l}c_{0}, \text { before } 17 \text { March } 2020 \\ \left(c_{0}-c_{b}\right) \mathrm{e}^{-r_{5}\left(t-T_{0}\right)}+c_{b}, \text { from } 17 \text { March to } 12 \text { June } 2020\end{array}\right.$

$\beta(t)=\left\{\begin{array}{l}\beta_{0}, \text { before } 17 \text { March } 2020 \\ \left(\beta_{0}-\beta_{a}\right) \mathrm{e}^{-r_{5}\left(t-T_{0}\right)}+\beta_{a}, \text { from } 17 \text { March to } 12 \text { June } 2020\end{array}\right.$

$q(t)=\left\{\begin{array}{l}q_{0}, \text { before } 17 \text { March } 2020 \\ \left(q_{0}-q_{m}\right) \mathrm{e}^{-r_{5}\left(t-T_{0}\right)}+q_{m}, \text { from } 17 \text { March to } 12 \text { June } 2020\end{array}\right.$

On the basis that the contact number increased by $100 \%$ (transmission probability and quarantined rate maintained the original trend), both contact number and transmission probability increased by $100 \%$ (quarantined rate maintained the original trend), and both contact number and transmission probability increased by $100 \%$ and quarantined rate contraction by $50 \%$, the risk of an outbreak in Singapore under all combinations of $k$ and $r_{5}$ was considered, as shown in Fig. 9 and Table 3.

As can be seen from Fig. 9 and Table 3, an outbreak of COVID-19 was possible and highly likely in Singapore. It is clear that changes in the contact number, transmission probability and quarantined rate and how quickly they change, significantly influence the extent of the outbreak. It is obvious that with the increasing proportion of undetected cases, the severity of the epi- the number of $H(t)$ would be close to 50,000 . And if the contact number was maintained, there would still be a secondary outbreak. Thus, in order to better analyse the relationship between the number of contacts and the secondary outbreak, we analysed the degree of the secondary outbreak caused by the number of contacts increased by $0,2 \%, 5 \%, 10 \%$ and $20 \%$, respectively, when $r_{5}=0.02$ and $k=0$ (as shown in Fig. 10).

Comparing Figs. 7 and 9, we find that even if the quarantined rate in Tianjin drops to a very low level, it is difficult to generate a secondary outbreak, and even if there is a secondary outbreak, the severity of the epidemic in Tianjin is negligible compared with that in Singapore. The importance of maintaining an effective reproduction number less than 1 is emphasized by our results, given our assumption that people in Tianjin started to go back to work and reopened schools 

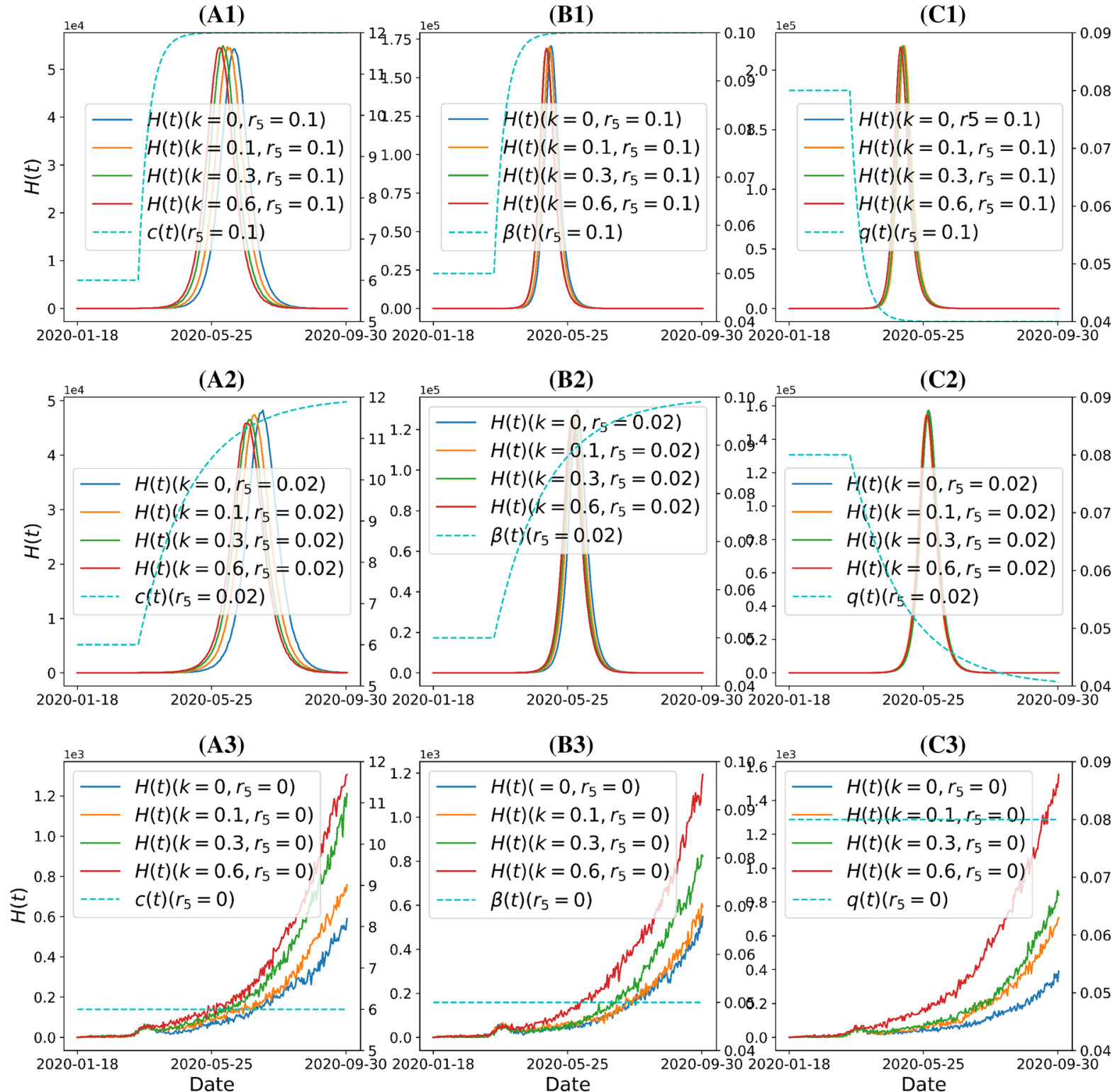

(B3)

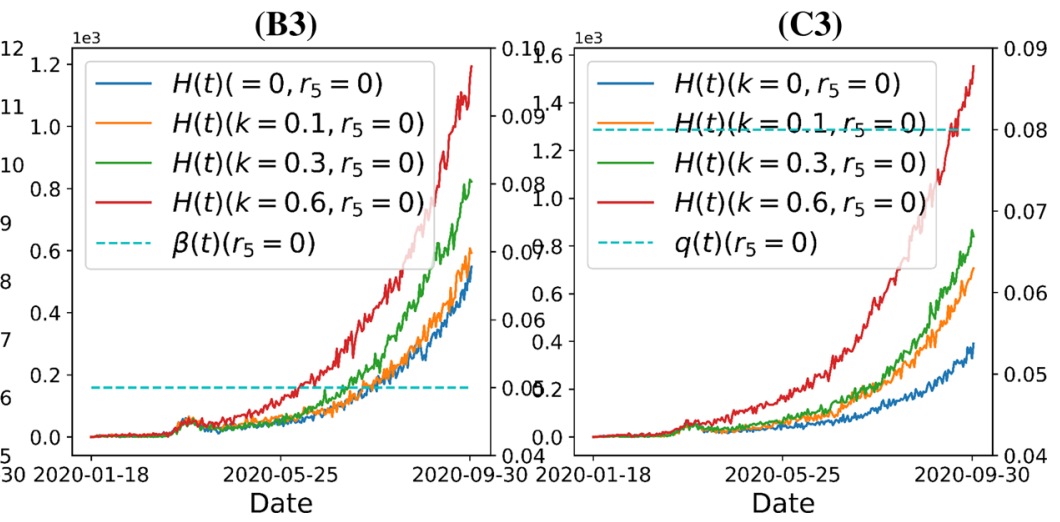

Fig. 9 Impact of foreign imported cases and lifting the prevention and control measures on the possibility of a secondary outbreak of COVID-19 in Singapore. A1-A3 The hospitalized cases based on contact number increased by $100 \%$ with all combinations of $k$ and $r_{5}$ and the corresponding $c(t)$. B1-B3 The hospitalized cases based on both contact number and transmis- sion probability increased by $100 \%$ with all combinations of $k$ and $r_{5}$ and the corresponding $\beta(t)$. C1-C3 The hospitalized cases based on both contact number and transmission probability increased by $100 \%$ and quarantined rate contraction by $50 \%$ with all combinations of $k$ and $r_{5}$ and the corresponding $q(t)$ 


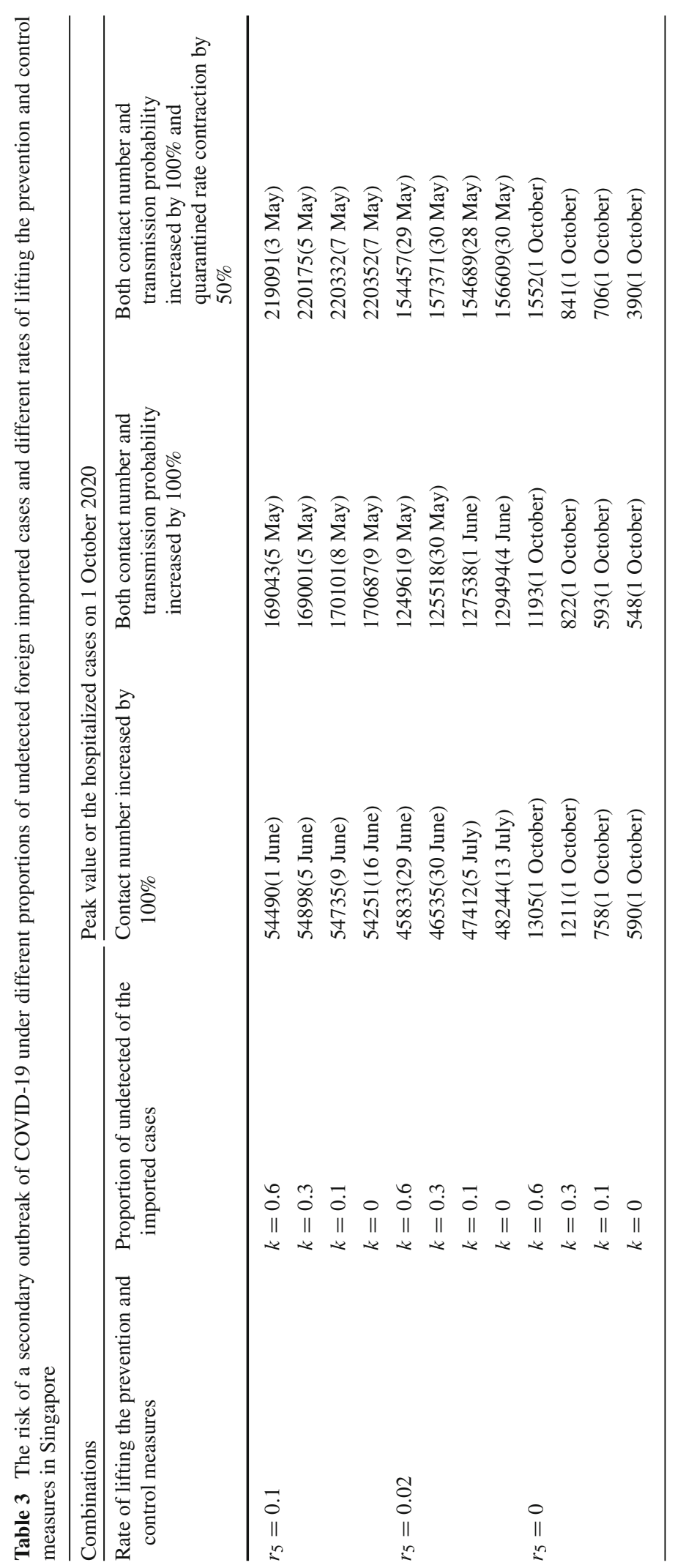




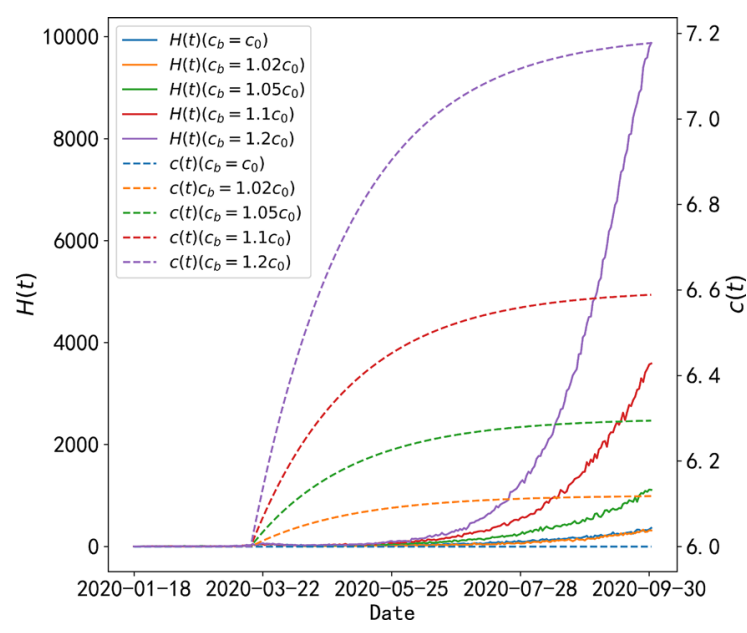

Fig. 10 Impacts of increasing the contact number on a potential secondary outbreak of COVID-19 with $k=0$ and $r_{5}=0.02$ in Singapore

with its effective reproduction number stable at around 0 for nearly a month and a half. Singapore, in contrast, started to ease restrictions when the effective reproduction number was greater than 1 . Therefore, if work was resumed and schools reopened with the effective reproduction number greater than 1 , the generation of a secondary outbreak would be more likely.

According to the Ministry of Health [12], the Prime Minister announced a set of strict measures to slow down the transmission of COVID-19 in Singapore in his address to the nation on 3 April. They imposed stringent measures from 7 April 2020 and introduced even stricter measures on 21 April 2020.

Therefore, 21 April is used as the turning point of new prevention and control measures. Combined with the assumption that various measures started to be loosened on 18 March, we get the corresponding changes of $c(t), \beta(t)$ and $q(t)$ as follows:
It is clear that formula (4) is the same as formula (3) for the period before 21 April, where the parameter $r_{5}=0.035$ except that the new parameter $r_{6}=1$ is introduced in the newly added prevention and control measures. The comparison between the simulation and the real data is shown in Fig. 11.

It can be seen from Fig. 11 that the weakening of prevention and control measures caused the secondary outbreak of the epidemic in Singapore. However, subsequent timely strengthening of the measures effectively controlled the worsening of the epidemic.

\section{Discussion and conclusions}

The COVID-19 epidemic in Tianjin after the Spring Festival was effectively controlled under strict prevention and control measures. Singapore kept its initial outbreak under control with strict measures taken from the start. However, after the two regions lifted their prevention and control measures, one had a secondary outbreak and the other had none. Two questions are raised: What are the key factors affecting the development of the epidemic? And when can we resume work and reopen schools? [29] To address these questions, we constructed a stochastic discrete dynamics model of COVID-19 based on epidemic data from Tianjin and Singapore.

In Tianjin, work began to resume and schools reopened after the effective reproduction number had been approaching 0 for one and a half months. We know that fully lifting the prevention and control measures about contact number $c$ and transmission possibility $\beta$ will not result in a secondary outbreak, and more than $60 \%$ lifting the prevention and control measures about quarantined rate $q$ will have a risk of secondary outbreak. Due to the effect of quarantine-related pre-

$$
\begin{aligned}
& c(t)=\left\{\begin{array}{l}
c_{0}, \text { before } 17 \text { March } 2020 \\
\left(c_{0}-c_{b}\right) \mathrm{e}^{-r_{5}\left(t-T_{0}\right)}+c_{b}, \text { from 17 March to 20 April } 2020 \\
\left(c_{01}-c_{b 1}\right) \mathrm{e}^{-r_{6}\left(t-T_{1}\right)}+c_{b 1}, \text { from 21 April to 12 June } 2020
\end{array}\right. \\
& \beta(t)=\left\{\begin{array}{l}
\beta_{0}, \text { before } 17 \text { March } 2020 \\
\left(\beta_{0}-\beta_{a}\right) \mathrm{e}^{-r_{5}\left(t-T_{0}\right)}+\beta_{a}, \text { from 17 March to 20 April } 2020 \\
\left(\beta_{01}-\beta_{a 1}\right) \mathrm{e}^{-r_{6}\left(t-T_{1}\right)}+\beta_{a 1}, \text { from 21 April to 12 June } 2020
\end{array}\right. \\
& q(t)=\left\{\begin{array}{l}
q_{0}, \text { before } 17 \text { March } 2020 \\
\left(q_{0}-q_{m}\right) \mathrm{e}^{-r_{5}\left(t-T_{0}\right)}+q_{m}, \text { from 17 March to 20 April } 2020 \\
\left(q_{01}-q_{m 1}\right) \mathrm{e}^{-r_{6}\left(t-T_{1}\right)}+q_{m 1}, \text { from 21 April to 12 June 2020 }
\end{array}\right.
\end{aligned}
$$


Fig. 11 a The estimated and real numbers of hospitalized individuals in Singapore from 18 January to 12 June 2020 . b-e The corresponding trends for $c(t), \beta(t), q(t)$ and $R(t)$
(A)

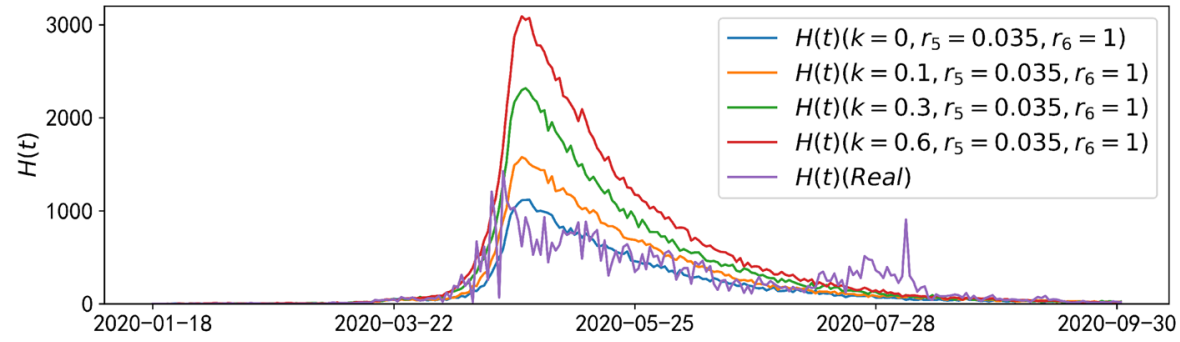

(B)

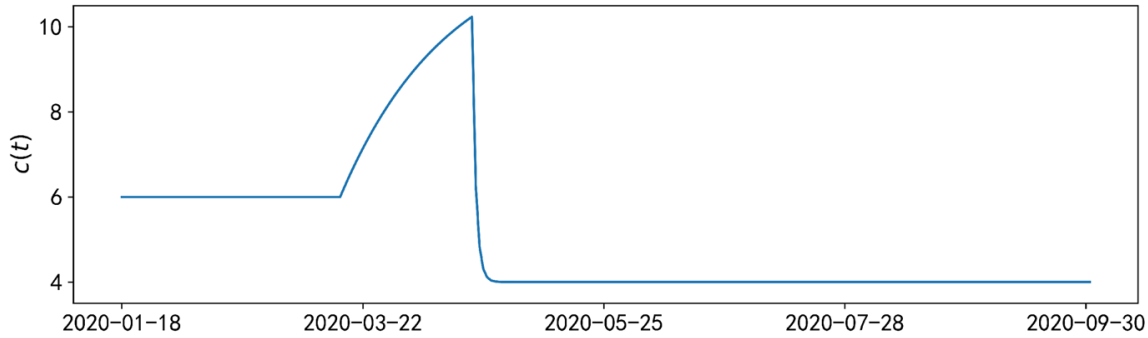

(C)

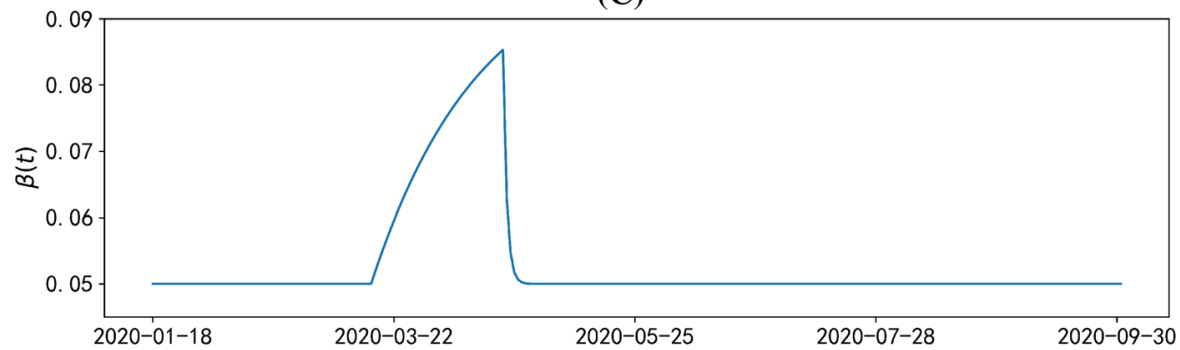

(D)

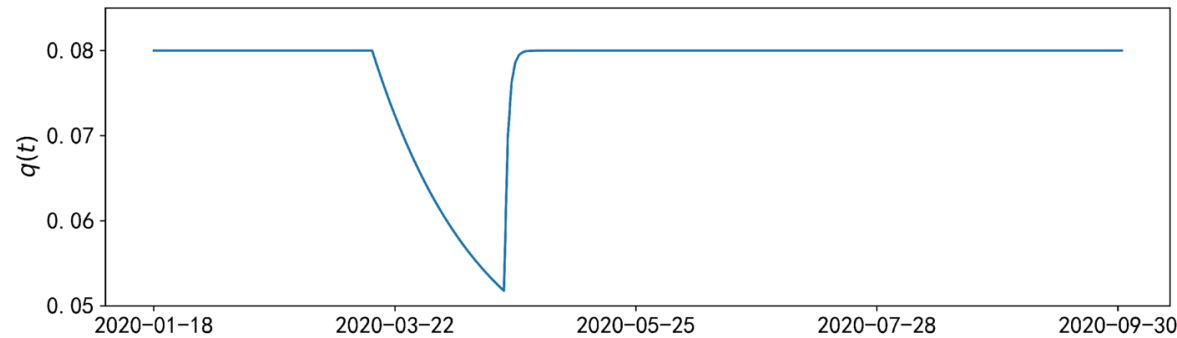

(E)

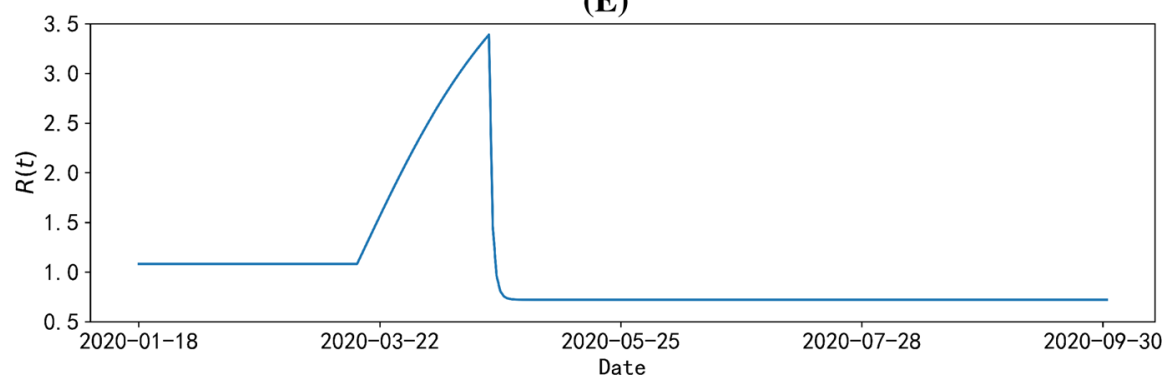


vention and control measures on the socioeconomics, it is advisable to keep a relative low quarantined rate, i.e. 0.16 at all times. For Singapore, the effective reproduction number was still more than 1 when it chose to return to resume work and reopen schools, and its quarantined rate was always low (less than 0.1). If its prevention and control measures are slightly relaxed, this will cause a secondary outbreak. The more relaxed the measures, the more serious the epidemic will be. In view of Singapore's low quarantined rate (with sufficient room for increase) and the effective reproduction number greater than 1 , it is recommended to increase the quarantined rate appropriately to make the effective reproduction number less than 1 before considering increasing the contact number [30].

The main conclusions show that strengthening the management of people entering the country and comprehensive tracing of close contacts when cases occur are key factors in preventing outbreaks. The time to resume work and reopen schools depends on the state of the local effective reproduction number. When the effective reproduction number is stable below 1 , it is better to keep it at 0 for a long time and maintain relatively strict prevention and control measures according to the above key factors. In this case, the possibility of a secondary outbreak of the epidemic will be relatively low if work and schooling resume. If there is a secondary outbreak, as long as close contacts can be controlled in a timely manner, the epidemic will be effectively controlled.

This paper has shown secondary outbreaks of different degrees caused by different extents to which control measures were lifted. But if we want to better develop strategies for COVID-19, medical resources also need to be considered. So one more compartment (confirmed but not hospitalized) should be introduced in model(1), to discuss how to ensure that the final size of the infected population is low and that the expenditure is economical, providing the train of thought for future research.

Acknowledgements This work was supported by the National Natural Science Foundation of China (NSFCs 12031010, 11631012, 61772017).

\section{Compliance with ethical standards}

Conflict of interest The authors declare that they have no conflict of interest.

\section{References}

1. Tianjin Bureau of Statistics. Available from: http://stats.tj. gov.cn/TJTJJ434/TJGK606/\#5 (accessed on 18 November 2020)

2. Ministry of Foreign Affairs of the People's Republic of China. Available from: https://www.fmprc.gov.cn/web/ (accessed on 18 November 2020)

3. Leung, K., Wu, J., Liu, D., Leung, G.M.: First-wave COVID19 transmissibility and severity in China outside Hubei after control measures, and second-wave scenario planning: a modelling impact assessment. Lancet (2020). https://doi. org/10.1016/S0140-6736(20)30746-7

4. $\mathrm{Xu}, \mathrm{S} ., \mathrm{Li}, \mathrm{Y}$. : Beware of the second wave of COVID-19. Lancet (2020). https://doi.org/10.1016/ S0140-6736(20)30845-X

5. Maier, B.F., Brockmann, D.: Effective containment explains subexponential growth in recent confirmed COVID-19 cases in China. Science 368(6492), 742-746 (2020)

6. Tian, H., Liu, Y., Li, Y., et al.: An investigation of transmission control measures during the first 50 days of the COVID19 epidemic in China. Science 368(6491), 638-642 (2020)

7. Saha, S., Samanta, G.P., Nieto, J.J.: Epidemic model of COVID-19 outbreak by inducing behavioural response in population. Nonlinear Dyn. 102(1), 455-487 (2020)

8. Niwa, M., Hara, Y., Sengoku, S., Kodama, K.: Effectiveness of Social Measures against COVID-19 Outbreaks in Selected Japanese Regions Analyzed by System Dynamic Modeling. Int. J. Environ. Res. Public Health 17(17),6238(2020)

9. Moradian, N., et al.: The urgent need for integrated science to fight COVID-19 pandemic and beyond. J. Transl. Med. 18(1), 205 (2020)

10. Upadhyay, R.K., Chatterjee, S., Saha, S., Azad, R.K.: Agegroup-targeted testing for covid-19 as a new prevention strategy. Nonlinear Dyn. 101(7798), 1921-1932 (2020)

11. Health Commission of Tianjin. Available from: http://wsjk.tj.gov.cn/ (accessed on 18 November 2020)

12. Ministry of Health of Singapore. Available from: https:// www.moh.gov.sg/ (accessed on 18 November 2020)

13. Zhou, Y., Ma, Z., Brauer, F.: A discrete epidemic model for SARS transmission and control in China. Math. Comput. Model. 40(13), 1491-1506 (2004)

14. Tang, S., Tang, B., Bragazzi, N.L., et al.: Analysis of COVID-19 epidemic traced data and stochastic discrete transmission dynamic model. Sci. Sin. Math. 50, 1-16 (2020)

15. Lekone, P.E., Finkenstadt, B.: Statistical inference in a stochastic epidemic SEIR model with control intervention: Ebola as a case study. Biometrics 62(4), 1170-1177 (2006)

16. Mode, C.J., Sleeman, C.K.: Stochastic processes in epidemiology: HIV/AIDS. Other infectious diseases and computers. World Scientific, Singapore (2000)

17. National Health Commission of the People's Republic of China. Available from: http://www.nhc.gov.cn (accessed on 18 November 2020)

18. Nishiura, H., Kobayashi, T., Miyama. T., et al.: Estimation of the asymptomatic ratio of novel coronavirus infections (COVID-19). International Journal of Infectious Diseases. 94, 154-155(2020) 
19. Wallinga, J., Teunis, P.: Different epidemic curves for severe acute respiratory syndrome reveal similar impacts of control measures. Am. J. Epidemiol. 160(6), 509-516 (2004)

20. Nishiura, H., Chowell, G.: The effective reproduction number as a prelude to statistical estimation of time-dependent epidemic trends. Math. Stat. Estimation Approac. Epidemiol. 2009, 103-121 (2009)

21. Li, X., Wang, W.: A discrete epidemic model with stage structure. Chaos Solitons Fractals. 26(3), 947-958 (2005)

22. Allen, L.J., Van den Driessche, P.: The basic reproduction number in some discrete-time epidemic models. J. Differ. Equ. Appl. 14(10-11), 1127-1147 (2008)

23. Bi, Q., Wu, Y., Mei, S., et al.: Epidemiology and transmission of COVID-19 in 391 cases and 1286 of their close contacts in Shenzhen, China: a retrospective cohort study. Lancet (2020). https://doi.org/10.1016/S1473-3099(20)30287-5

24. Zhang, J., Litvinova, M., Liang, Y., et al.: Changes in contact patterns shape the dynamics of the COVID-19 outbreak in China. Science 368(6498), 1481-1486 (2020)

25. Reddy, K.P., Shebl, F.M., Foote, J.H.A., et al.: Costeffectiveness of public health strategies for COVID-19 epidemic control in South Africa: a microsimulation modelling study. Lancet (2020). https://doi.org/10.1016/ S2214-109X(20)30452-6
26. Li, Y., Campbell, H., Kulkarni, D., et al.: The temporal association of introducing and lifting non-pharmaceutical interventions with the time-varying reproduction number (R) of SARS-CoV-2: a modelling study across 131 countries. Lancet (2020). https://doi.org/10.1016/ S1473-3099(20)30785-4

27. Haushofer, J., Metcalf, C.J.E.: Which interventions work best in a pandemic? Science 368(6495), 1063-1065 (2020)

28. Beijing Municipal Health Commission. Available from: http://wjw.beijing.gov.cn/ (accessed on 18 November 2020)

29. Lordan, R., Fitzgerald, G.A., Grosser, T.: Reopening schools during COVID-19. Science 369(3508), 1146 (2020)

30. Alwan, N.A., Burgess, R.A., Ashworth, S., et al.: Scientific consensus on the COVID-19 pandemic: we need to act now. Lancet (2020). https://doi.org/10.1016/ S0140-6736(20)32153-X

Publisher's Note Springer Nature remains neutral with regard to jurisdictional claims in published maps and institutional affiliations. 